\title{
Generalized $q$-Bessel function and its properties
}

Mansour Mahmoud*

"Correspondence:
mansour@mans.edu.eg
Mathematics Department, Faculty
of Science, King Abdulaziz
University, P.O. Box 80203, Jeddah,
21589, Saudi Arabia
Permanent address: Department of
Mathematics, Faculty of Science,
Mansoura University, Mansoura,
35516, Egypt

"Correspondence:

mansour@mans.edu.eg

of Science, King Abdulaziz

University, P.O. Box 80203, Jeddah,

35516, Egypt

\begin{abstract}
In this paper, the generalized $q$-Bessel function, which is a generalization of the known $q$-Bessel functions of kinds 1,2,3, and the new q-analogy of the modified Bessel function presented in (Mansour and Al-Shomarani in J. Comput. Anal. Appl. 15(4):655-664, 2013) is introduced. We deduced its generating function, recurrence relations and $q$-difference equation, which gives us the differential equation of each of the Bessel function and the modified Bessel function when $q$ tends to 1. Finally, the quantum algebra $E_{2}(q)$ and its representations presented an algebraic derivation for the generating function of the generalized $q$-Bessel function.
\end{abstract}

MSC: Primary 33D45; 81R50; 22E70

Keywords: $q$-Bessel functions; generating function; $q$-difference equation; quantum algebra; irreducible representation

\section{Introduction}

The $q$-shifted factorials are defined by [1]

$$
\begin{aligned}
& (a ; q)_{0}=1, \\
& (a ; q)_{k}=\prod_{i=0}^{k-1}\left(1-a q^{k}\right), \\
& \left(a_{1}, \ldots, a_{r} ; q\right)_{k}=\prod_{j=1}^{r}\left(a_{j} ; q\right)_{k} ; \quad k=0,1,2, \ldots, \\
& (a ; q)_{\infty}=\prod_{i=0}^{\infty}\left(1-a q^{i}\right), \quad \text { where } a, a_{i}{ }^{\prime} \mathrm{s}, q \in \mathbb{R} \text { such that } 0<q<1 .
\end{aligned}
$$

The one-parameter family of $q$-exponential functions

$$
E_{q}^{(\alpha)}(z)=\sum_{n=0}^{\infty} \frac{q^{\frac{\alpha n^{2}}{2}}}{(q ; q)_{n}} z^{n}
$$

with $\alpha \in \mathbb{R}$ has been considered in [2]. Consequently, in the limit when $q \rightarrow 1$, we have $\lim _{q \rightarrow 1} E_{q}^{(\alpha)}((1-q) z)=e^{z}$. Exton [3] presented the following $q$-exponential functions:

$$
E(\mu, z ; q)=\sum_{n=0}^{\infty} \frac{q^{\mu n(n-1)}}{[n]_{q} !} z^{n},
$$

(0) 2013 Mahmoud; licensee Springer. This is an Open Access article distributed under the terms of the Creative Commons Attribution License (http://creativecommons.org/licenses/by/2.0), which permits unrestricted use, distribution, and reproduction in any medium, provided the original work is properly cited. 
where $[n]_{q} !=\frac{(q ; q)_{n}}{(1-q)^{n}}$. The relation between these two notations is given by

$$
E(\lambda, z ; q)=E_{q}^{(2 \lambda)}\left(q^{-\lambda}(1-q) z\right)
$$

In Exton's formula, if we replace $z$ by $\frac{x}{1-q}$ and $\mu$ by $2 a$, we get the following $q$-exponential function:

$$
E_{q}(x, a)=\sum_{n=0}^{\infty} \frac{q^{a\left(\begin{array}{c}
n \\
2
\end{array}\right)}}{(q ; q)_{n}} x^{n}
$$

which satisfies the functional relation [4]

$$
E_{q}(x, a)-E_{q}(q x, a)=x E_{q}\left(q^{a} x, a\right)
$$

which can be rewritten by the formula

$$
D_{q} E_{q}(x, a)=\frac{1}{1-q} E_{q}\left(q^{a} x, a\right),
$$

where the Jackson $q$-difference operator $D_{q}$ is defined by [5]

$$
D_{q} f(x)=\frac{f(x)-f(q x)}{(1-q) x}
$$

and satisfies the product rule

$$
D_{q}(f(x) g(x))=f(q x) D_{q} g(x)+g(x) D_{q} f(x) .
$$

There are two important special cases of the function $E_{q}(x, a)$

$$
e_{q}(x)=\sum_{n=0}^{\infty} \frac{x^{n}}{(q, q)_{n}}, \quad|x|<1
$$

and

$$
E_{q}(x)=\sum_{n=0}^{\infty} \frac{q^{\left(\begin{array}{c}
n \\
2
\end{array}\right)} x^{n}}{(q, q)_{n}} .
$$

The $q$-Bessel functions of kinds 1, 2 and 3 are defined by [6]

$$
\begin{aligned}
& J_{n}^{(1)}(x ; q)=\frac{\left(q^{n+1} ; q\right)_{\infty}}{(q ; q)_{\infty}}\left(\frac{x}{2}\right)^{n}{ }_{2} \varphi_{1}\left(\begin{array}{c|c}
0,0 \\
q^{n+1}
\end{array} \mid q ;-\frac{x^{2}}{4}\right), \\
& J_{n}^{(2)}(x ; q)=\frac{\left(q^{n+1} ; q\right)_{\infty}}{(q ; q)_{\infty}}\left(\frac{x}{2}\right)^{n}{ }_{0} \varphi_{1}\left(\begin{array}{c|c}
- & q ;-\frac{q^{n+1} x^{2}}{4} \\
q^{n+1} & q
\end{array}\right. \\
& J_{n}^{(3)}(x ; q)=\frac{\left(q^{n+1} ; q\right)_{\infty}}{(q ; q)_{\infty}}\left(\frac{x}{2}\right)^{n}{ }_{1} \varphi_{1}\left(\begin{array}{c|c}
0 & q ;-\frac{q^{\frac{n+1}{2}} x^{2}}{4}
\end{array} q^{n+1} \mid\right.
\end{aligned}
$$


where ${ }_{r} \varphi_{s}$ is the basic hypergeometric function [1]

$$
{ }_{r} \varphi_{s}\left(\begin{array}{c}
a_{1}, \ldots, a_{r} \\
b_{1}, \ldots, b_{s}
\end{array} \mid q ; z\right)=\sum_{k=0}^{\infty} \frac{\left(a_{1}, \ldots, a_{r} ; q\right)_{k}}{\left(b_{1}, \ldots, b_{s} ; q\right)_{k}}\left((-1)^{k} q^{\frac{k}{2}(k-1)}\right)^{1+s-r} \frac{z^{k}}{(q ; q)_{k}} .
$$

The functions $J_{n}^{(i)}(x ; q), i=1,2$, are $q$-analogues of the Bessel function, and the function $J_{n}^{(3)}(x ; q)$ is a $q$-analogue of the modified Bessel function.

Rogov $[7,8]$ introduced generalized modified $q$-Bessel functions, similarly to the classical case [9], as

$$
I_{n}^{i}(x ; q)=\frac{\left(q^{n+1} ; q\right)_{\infty}}{(q ; q)_{\infty}}\left(\frac{x}{2}\right)^{n}{ }_{\delta} \varphi_{1}\left(\begin{array}{c}
0,0, \ldots, 0 \\
q^{n+1}
\end{array} \mid q ; \frac{q^{\frac{(n+1)(2-\delta)}{2}} x^{2}}{4}\right),
$$

where

$$
\delta= \begin{cases}2 & \text { for } i=1, \\ 0 & \text { for } i=2, \\ 1 & \text { for } i=3 .\end{cases}
$$

Recently, Mansour and et al. [10] studied the following $q$-Bessel function:

$$
J_{n}^{(4)}(x ; q)=\frac{(x / 2)^{n}}{(q ; q)_{n}} 0 \varphi_{2}\left(\begin{array}{c|c}
- & q ; q^{n+1} \\
0, \frac{3(n+1)}{2} x^{2} & 4
\end{array}\right)
$$

which is a $q$-analogy of the modified Bessel function.

In this paper, we define the generalized $q$-Bessel function and study some of its properties. Also, in analogy with the ordinary Lie theory $[11,12]$, we derive algebraically the generating function of the generalized $q$-Bessel function.

\section{The generalized $q$-Bessel function and its generating function}

Definition 2.1 The generalized $q$-Bessel function is defined by

$$
J_{n}(x, a ; q)=\frac{(x / 2)^{n}}{(q ; q)_{n}} \sum_{k=0}^{\infty} \frac{(-1)^{k(a+1)} q^{\frac{a k}{2}(k+n)}}{\left(q^{n+1} ; q\right)_{k}} \frac{\left(x^{2} / 4\right)^{k}}{(q ; q)_{k}}
$$

which converges absolutely for all $x$ when $a \in \mathbb{Z}^{+}$and for $|x|<2$ if $a=0$.

As special cases of $J_{n}(x, a ; q)$, we get

$$
\begin{aligned}
& J_{n}^{(1)}(x ; q)=J_{n}(x, 0 ; q), \\
& J_{n}^{(2)}(x ; q)=J_{n}(x, 2 ; q), \\
& J_{n}^{(3)}(x ; q)=J_{n}(x, 1 ; q), \\
& J_{n}^{(4)}(x ; q)=J_{n}(x, 3 ; q) .
\end{aligned}
$$

Lemma 2.2 The function $J_{n}(x, a ; q)$ is a q-analogy of each of the Bessel function and the modified Bessel function. 
Proof

$$
\begin{aligned}
\lim _{q \rightarrow 1} J_{n}((1-q) x, a ; q) & =\lim _{q \rightarrow 1}\left\{\frac{(1-q)^{n}}{(q, q)_{n}}\left(\frac{x}{2}\right)^{n} \sum_{k=0}^{\infty}(-1)^{k(a+1)} q^{\frac{a k(k+n)}{2}} \frac{(1-q)^{2 k}}{\left(q^{n+1} ; q\right)_{k}(q ; q)_{k}}\left(\frac{x}{2}\right)^{2 k}\right\} \\
& =\frac{1}{(1)_{n}}\left(\frac{x}{2}\right)^{n} \sum_{k=0}^{\infty} \frac{(-1)^{k(a+1)}}{(n+1)_{k}(1)_{k}}\left(\frac{x}{2}\right)^{2 k} \\
& =\left(\frac{x}{2}\right)^{n} \sum_{k=0}^{\infty} \frac{(-1)^{k(a+1)}}{\Gamma(n+k+1) \Gamma(k+1)}\left(\frac{x}{2}\right)^{2 k} .
\end{aligned}
$$

Hence, we get

$$
\lim _{q \rightarrow 1} J_{n}((1-q) x, a ; q)=J_{n}(x) ; \quad a=0,2,4, \ldots
$$

and

$$
\lim _{q \rightarrow 1} J_{n}((1-q) x, a ; q)=I_{n}(x) ; \quad a=1,3,5, \ldots
$$

where $J_{n}(x)$ is the Bessel function and $I_{n}(x)$ is the modified Bessel function.

Lemma 2.3 The function $J_{n}(x, a ; q)$ satisfies

$$
J_{-n}(x, a ; q)=(-1)^{n(a+1)} J_{n}(x, a ; q), \quad n \in \mathbb{Z} .
$$

Proof Using the definition (10), we get

$$
J_{-n}(x, a ; q)=\sum_{k=n}^{\infty} \frac{(-1)^{k(a+1)} q^{\frac{a k(k-n)}{2}}\left(q^{-n+k+1} ; q\right)_{\infty}}{(q ; q)_{\infty}(q ; q)_{k}}\left(\frac{x}{2}\right)^{2 k-n}
$$

For $s=k-n$, we obtain

$$
J_{-n}(x, a ; q)=\sum_{s=0}^{\infty} \frac{(-1)^{(s+n)(a+1)} q^{\frac{a s(s+n)}{2}}\left(q^{s+1} ; q\right)_{\infty}}{(q ; q)_{\infty}(q ; q)_{s+n}}\left(\frac{x}{2}\right)^{2 s+n}
$$

and using the relations [1]

$$
\begin{aligned}
& \left(q^{s+1} ; q\right)_{\infty}=\left(q^{n+s+1} ; q\right)_{\infty}\left(q^{s+1} ; q\right)_{n}, \\
& (q ; q)_{s+n}=(q ; q)_{s}\left(q^{s+1} ; q\right)_{n}
\end{aligned}
$$

we obtain

$$
\begin{aligned}
J_{-n}(x, a ; q) & =(-1)^{n(a+1)} \sum_{s=0}^{\infty} \frac{(-1)^{s(a+1)} q^{\frac{a s(s+n)}{2}}\left(q^{n+s+1} ; q\right)_{\infty}}{(q ; q)_{\infty}(q ; q)_{s}}\left(\frac{x}{2}\right)^{2 s+n} \\
& =(-1)^{n(a+1)} J_{n}(x, a ; q) .
\end{aligned}
$$


Lemma 2.4 The function $J_{n}(x, a ; q)$ satisfies the relation

$$
J_{n}(-x, a ; q)=(-1)^{n} J_{n}(x, a ; q), \quad n \in \mathbb{Z},
$$

and hence it is even (or odd) function if the integer $n$ is even (or odd).

Now we will deduce the generating function of the generalized $q$-Bessel function $J_{n}(x, a ; q)$.

Theorem 1 The generating function $g(x, t, a ; q)$ of the function $J_{n}(x, a ; q)$ is given by

$$
g(x, t, a ; q)=E_{q}\left(\frac{q^{\frac{a}{4}} x t}{2}, \frac{a}{2}\right) E_{q}\left(\frac{(-1)^{a+1} q^{\frac{a}{4}} x}{2 t}, \frac{a}{2}\right)=\sum_{n=-\infty}^{\infty} q^{\frac{a n^{2}}{4}} J_{n}(x, a ; q) t^{n} .
$$

Proof Let

$$
g(x, t, a ; q)=E_{q}\left(\frac{q^{\frac{a}{4}} x t}{2}, \frac{a}{2}\right) E_{q}\left(\frac{(-1)^{a+1} q^{\frac{a}{4}} x}{2 t}, \frac{a}{2}\right),
$$

then

$$
g(x, t, a ; q)=\sum_{r=0}^{\infty} \sum_{s=0}^{\infty} \frac{(-1)^{s(a+1)} q^{\frac{a}{2}\left[\left(\begin{array}{l}
r \\
2
\end{array}\right)+\left(\begin{array}{c}
s \\
2
\end{array}\right)\right]+\frac{a}{4}(s+r)}}{(q ; q)_{r}(q ; q)_{s}}\left(\frac{x}{2}\right)^{s+r} t^{r-s} .
$$

For $s=r-n$, we get

$$
g(x, t, a ; q)=\sum_{n=-\infty}^{\infty} \sum_{r=0}^{\infty} \frac{(-1)^{(r-n)(a+1)} q^{\left.\frac{a}{2}\left[\begin{array}{c}
r \\
2
\end{array}\right)+\left(\begin{array}{c}
r-n \\
2
\end{array}\right)\right]+\frac{a}{4}(2 r-n)}}{(q ; q)_{r}(q ; q)_{r-n}}\left(\frac{x}{2}\right)^{2 r-n} t^{n} .
$$

Hence, for $n \leq 0$, the coefficient of $t^{n}$ is given by

$$
c_{n}=(-1)^{-n(a+1)} q^{\frac{a n^{2}}{4}} \frac{(x / 2)^{-n}}{(q ; q)_{-n}} \sum_{r=0}^{\infty} \frac{(-1)^{r(a+1)} q^{\frac{a r}{2}(r-n)}}{(q ; q)_{r}\left(q^{-n+1} ; q\right)_{r}}\left(\frac{x}{2}\right)^{2 r},
$$

where $(q, q)_{r-n}=(q ; q)_{-n}\left(q^{-n+1} ; q\right)_{r}$ for $n \leq 0$. Then

$$
c_{n}=(-1)^{-n(a+1)} q^{\frac{a n^{2}}{4}} J_{-n}(x, a ; q)=q^{\frac{a n^{2}}{4}} J_{n}(x, a ; q) .
$$

Similarly, for $n \geq 0$.

As special cases of $g_{n}(x, t, a ; q)$, we obtain

$$
g_{n}(x, t, 0 ; q)=E_{q}\left(\frac{x t}{2}, 0\right) E_{q}\left(\frac{-x}{2 t}, 0\right)=e_{q}\left(\frac{x t}{2}\right) e_{q}\left(\frac{-x}{2 t}\right),
$$

which is a generating function of the $q$-Bessel function $J_{n}^{(1)}(x ; q)$ [13],

$$
g_{n}\left(x, \frac{t}{\sqrt{q}}, 2 ; q\right)=E_{q}\left(\frac{x t}{2}, 1\right) E_{q}\left(\frac{-q x}{2 t}, 1\right)=E_{q}\left(\frac{x t}{2}\right) E_{q}\left(\frac{-q x}{2 t}\right),
$$


which is a generating function of the $q$-Bessel function $J_{n}^{(2)}(x ; q)$ [13],

$$
g_{n}(x, t, 1 ; q)=E_{q}\left(\frac{\sqrt[4]{q} x t}{2}, \frac{1}{2}\right) E_{q}\left(\frac{\sqrt[4]{q} x}{2 t}, \frac{1}{2}\right)
$$

which is a generating function of the $q$-Bessel function $J_{n}^{(3)}(x ; q)$ and

$$
g_{n}(x, t, 3 ; q)=E_{q}\left(\frac{q^{3 / 4} x t}{2}, \frac{3}{2}\right) E_{q}\left(\frac{q^{3 / 4} x}{2 t}, \frac{3}{2}\right),
$$

which is a generating function of the $q$-Bessel function $J_{n}^{(4)}(x ; q)[10]$.

\section{The $q$-difference equation of the function $J_{n}(x, a ; q)$}

Now the generating function method [13] will be used to deduce the $q$-difference equation of the generalized $q$-Bessel function. Using equation (15), we have

$$
E_{q}\left(\frac{q^{\frac{a}{4}} x t h}{2}, \frac{a}{2}\right) E_{q}\left(\frac{(-1)^{a+1} q^{\frac{a}{4}} x h}{2 t}, \frac{a}{2}\right)=\sum_{n=-\infty}^{\infty} q^{\frac{a n^{2}}{4}} J_{n}(x h, a ; q) t^{n} ; \quad h \in \mathbb{R}-\{0\} .
$$

By applying the operator $D_{q}$, we get

$$
\begin{aligned}
& \frac{(-1)^{a+1} q^{\frac{a}{4}} h}{2(1-q) t} E_{q}\left(\frac{q^{\frac{a+4}{4}} x t h}{2}, \frac{a}{2}\right) E_{q}\left(\frac{(-1)^{a+1} q^{\frac{3 a}{4}} x h}{2 t}, \frac{a}{2}\right) \\
& \quad+\frac{q^{\frac{a}{4}} t h}{2(1-q)} E_{q}\left(\frac{q^{\frac{3 a}{4}} x t h}{2}, \frac{a}{2}\right) E_{q}\left(\frac{(-1)^{a+1} q^{\frac{a}{4}} x h}{2 t}, \frac{a}{2}\right) \\
& =\sum_{n=-\infty}^{\infty} q^{\frac{a n^{2}}{4}} D_{q} J_{n}(x h, a ; q) t^{n} .
\end{aligned}
$$

Using equation (20), we obtain

$$
\begin{aligned}
& \frac{(-1)^{a+1} q^{\frac{a}{4}+\frac{a}{2}\left(\begin{array}{c}
n+1 \\
2
\end{array}\right)+\frac{n+1}{2}}}{2(1-q)} J_{n+1}\left(q^{\frac{a+2}{4}} x h, a ; q\right)+\frac{q^{\frac{a}{4}+\frac{a n(n-1)}{4}}}{2(1-q)} J_{n-1}\left(q^{\frac{a}{4}} x h, a ; q\right) \\
& =\frac{q^{\frac{a n^{2}}{4}}}{h} D_{q} J_{n}(x h, a ; q) \text {. }
\end{aligned}
$$

Hence

$$
\begin{aligned}
& D_{q} J_{n}(x h, a ; q) \\
& \quad=\frac{q^{\frac{a}{4}} h}{2(1-q)}\left\{(-1)^{a+1} q^{\frac{n(a+2)+2}{4}} J_{n+1}\left(q^{\frac{a+2}{4}} x h, a ; q\right)+q^{\frac{-a n}{4}} J_{n-1}\left(q^{\frac{a}{4}} x h, a ; q\right)\right\} .
\end{aligned}
$$

Similarly, we can prove the following relation:

$$
\begin{aligned}
& D_{q} J_{n}(x h, a ; q) \\
& \quad=\frac{q^{\frac{a}{4}} h}{2(1-q)}\left\{(-1)^{a+1} q^{\frac{n a}{4}} J_{n+1}\left(q^{\frac{a}{4}} x h, a ; q\right)+q^{\frac{(-a n-2 n+2)}{4}} J_{n-1}\left(q^{\frac{a+2}{4}} x h, a ; q\right)\right\} .
\end{aligned}
$$


By using equations (21) and (22), we obtain

$$
\begin{aligned}
& (-1)^{a+1} q^{\frac{n(a+1)+1}{2}} J_{n+1}(\sqrt{q} x h, a ; q)+J_{n-1}(x h, a ; q) \\
& =(-1)^{(a+1)} q^{\frac{a n}{2}} J_{n+1}(x h, a ; q)+q^{\frac{1-n}{2}} J_{n-1}(\sqrt{q} x h, a ; q) .
\end{aligned}
$$

But

$$
\begin{aligned}
J_{n-1}(\sqrt{q} x h, a ; q)= & \frac{\left(\frac{\sqrt{q} x h}{2}\right)^{n-1}}{(q ; q)_{\infty}} \sum_{k=0}^{\infty} \frac{(-1)^{k(a+1)} q^{\frac{a k}{2}(k+n-1)}\left(q^{n+k} ; q\right)_{\infty}}{(q ; q)_{k}}\left(\frac{\sqrt{q} x h}{2}\right)^{2 k} \\
= & \frac{q^{\frac{n-1}{2}}\left(\frac{x h}{2}\right)^{n-1}}{(q ; q)_{\infty}} \sum_{k=0}^{\infty} \frac{(-1)^{k(a+1)} q^{\frac{a k}{2}(k+n-1)}\left(q^{n+k} ; q\right)_{\infty}}{(q ; q)_{k}}\left(q^{k}-1+1\right)\left(\frac{x h}{2}\right)^{2 k} \\
= & q^{\frac{n-1}{2}} J_{n-1}(x h, a ; q)-\frac{q^{\frac{n-1}{2}\left(\frac{x h}{2}\right)^{n-1}}}{(q ; q)_{\infty}} \sum_{k=0}^{\infty} \frac{(-1)^{(k+1)(a+1)} q^{\frac{a(k+1)(k+n)}{2}}}{(q ; q)_{k}} \\
& \times\left(q^{n+k+1} ; q\right)_{\infty}\left(\frac{x h}{2}\right)^{2 k+2} \\
= & q^{\frac{n-1}{2}}\left\{J_{n-1}(x h, a ; q)+\frac{(-1)^{a} q^{\frac{a n}{2}} x h}{2} J_{n}\left(q^{\frac{a}{4}} x h, a ; q\right)\right\} .
\end{aligned}
$$

Then

$$
q^{\frac{1-n}{2}} J_{n-1}(\sqrt{q} x h, a ; q)-J_{n-1}(x h, a ; q)=\frac{(-1)^{a} q^{\frac{a n}{2}} x h}{2} J_{n}\left(q^{\frac{a}{4}} x h, a ; q\right) .
$$

Equations (23) and (24) give the relation

$$
q^{\frac{1+n}{2}} J_{n+1}(\sqrt{q} x h, a ; q)-J_{n+1}(x h, a ; q)=\frac{-x h}{2} J_{n}\left(q^{\frac{a}{4}} x h, a ; q\right) .
$$

Equations (21) and (25) give the relation

$$
\left\{D_{q}+\frac{q^{\frac{a(1-n)}{4}}}{(1-q) x}\left[q^{\frac{n}{2}} \delta_{q}-1\right]\right\} J_{n}(x h, a ; q)=\frac{(-1)^{a+1} h q^{\frac{(a+2)(n+1)}{4}}}{2(1-q)} J_{n+1}\left(q^{\frac{a+2}{4}} x h, a ; q\right),
$$

where the operator $\delta_{q}$ is given by $\delta_{q} f(x)=f(\sqrt{q} x)$.

Now consider the following operator:

$$
M_{n, q}=\left\{D_{q}+\frac{q^{\frac{a(1-n)}{4}}}{(1-q) x}\left[q^{\frac{n}{2}} \delta_{q}-1\right]\right\}
$$

then we can rewrite equation (26) by the formula

$$
M_{n, q} J_{n}(x h, a ; q)=\frac{(-1)^{a+1} h q^{\frac{(a+2)(n+1)}{4}}}{2(1-q)} J_{n+1}\left(q^{\frac{a+2}{4}} x h, a ; q\right) .
$$

Also, equations (22) and (24) give the relation

$$
\left\{D_{q}+\frac{q^{\frac{-a(1+n)}{4}}}{(1-q) x}\left[q^{\frac{-n}{2}} \delta_{q}-1\right]\right\} J_{n}(x h, a ; q)=\frac{h q^{\frac{(a+2)(1-n)}{4}}}{2(1-q)} J_{n-1}\left(q^{\frac{a+2}{4}} x h, a ; q\right) .
$$


If we consider the operator

$$
N_{n, q}=\left\{D_{q}+\frac{q^{\frac{-a(1+n)}{4}}}{(1-q) x}\left[q^{\frac{-n}{2}} \delta_{q}-1\right]\right\}
$$

then we can rewrite equation (29) by the formula

$$
N_{n, q} J_{n}(x h, a ; q)=\frac{h q^{\frac{(a+2)(1-n)}{4}}}{2(1-q)} J_{n-1}\left(q^{\frac{a+2}{4}} x h, a ; q\right) .
$$

Hence, the $q$-difference equation of the function $J_{n}(x, a ; q)$ takes the formula

$$
M_{n-1, q} N_{n, q} J_{n}(x h, a ; q)=\frac{(-1)^{a+1} q^{\frac{a+2}{4}} h^{2}}{4(1-q)^{2}} J_{n}\left(q^{\frac{a+2}{2}} x h, a ; q\right) .
$$

If we replace $h$ by $1-q$ and consider the limit as $q$ tends to 1 , then we obtain

$$
\left(\frac{1}{2} \frac{d}{d x}-\frac{n-1}{2 x}\right)\left(\frac{1}{2} \frac{d}{d x}+\frac{n}{2 x}\right) y(x)=\frac{(-1)^{a+1}}{4} y(x),
$$

or

$$
x^{2} y^{\prime \prime}(x)+x y^{\prime}-\left(n^{2}+(-1)^{a+1} x^{2}\right) y=0 .
$$

The differential equation (34) gives the Bessel function at $a=0,2,4, \ldots$ and the modified Bessel function at $a=1,3,5, \ldots$, which proves again that $J_{n}(x, a ; q)$ is a $q$-analogy of each of them.

4 The recurrence relations of the function $J_{n}(x, a ; q)$

\section{Lemma 4.1}

$$
J_{n}(x, a ; q)=\frac{2}{x}\left(1-q^{n+1}\right) J_{n+1}\left(q^{-a / 4} x, a ; q\right)+(-1)^{a+1} q^{\frac{(a+2)(n+1)}{2}} J_{n+2}(x, a ; q) .
$$

Proof

$$
\begin{aligned}
J_{n}(x, a ; q)= & \frac{(x / 2)^{n}}{(q ; q)_{n}} \sum_{k=0}^{\infty} \frac{(-1)^{k(a+1)} q^{\frac{a k}{2}(k+n)}}{(q ; q)_{k}\left(q^{n+1} ; q\right)_{k+1}}\left(1-q^{n+1}+q^{n+1}-q^{n+k-1}\right)\left(\frac{x^{2}}{4}\right)^{k} \\
= & \frac{2}{x}\left(1-q^{n+1}\right) \frac{(x / 2)^{n+1}}{(q ; q)_{n+1}} \sum_{k=0}^{\infty} \frac{(-1)^{k(a+1)} q^{\frac{a k}{2}(k+n+1)}}{(q ; q)_{k}\left(q^{n+2} ; q\right)_{k}}\left(\frac{\left(q^{-\frac{a}{4}} x\right)^{2}}{4}\right)^{k} \\
& +q^{n+1} \frac{(x / 2)^{n+2}}{(q ; q)_{n+2}} \sum_{k=1}^{\infty} \frac{(-1)^{k(a+1)} q^{\frac{a k}{2}(k+n)}}{(q ; q)_{k-1}\left(q^{n+3} ; q\right)_{k-1}}\left(\frac{x^{2}}{4}\right)^{k-1} \\
= & \frac{2}{x}\left(1-q^{n+1}\right) J_{n+1}\left(q^{-a / 4} x, a ; q\right) \\
& +(-1)^{a+1} q^{\frac{(a+2)(n+1)}{2}} \frac{(x / 2)^{n+2}}{(q ; q)_{n+2}} \sum_{k=0}^{\infty} \frac{(-1)^{k(a+1)} q^{\frac{a k}{2}(k+n+2)}}{(q ; q)_{k}\left(q^{n+3} ; q\right)_{k}}\left(\frac{x^{2}}{4}\right)^{k} \\
= & \frac{2}{x}\left(1-q^{n+1}\right) J_{n+1}\left(q^{-a / 4} x, a ; q\right)+(-1)^{a+1} q^{\frac{(a+2)(n+1)}{2}} J_{n+2}(x, a ; q) .
\end{aligned}
$$


Similarly, if we write $\left(1-q^{k}+q^{k}-q^{n+k-1}\right)$ instead of $\left(1-q^{n+1}+q^{n+1}-q^{n+k-1}\right)$, we can prove the following lemma.

\section{Lemma 4.2}

$$
J_{n}(x, a ; q)=\frac{2}{x}\left(1-q^{n+1}\right) J_{n+1}\left(q^{\frac{2-a}{4}} x, a ; q\right)+(-1)^{a+1} q^{\frac{a(n+1)}{2}} J_{n+2}(x, a ; q) .
$$

Now, if we replace $a$ by $a+2$ in the recurrence relation (36), we get the recurrence relation (35). Then we have the following lemma.

Lemma 4.3 The two functions $J_{n}(x, a ; q)$ and $J_{n}(x, a+2 ; q)$ have the same recurrence relation.

Then we have two cases of the recurrence relation.

Case (1): The function $J_{n}(x, a ; q)$ has the recurrence relation

$$
J_{n}(x, a ; q)=\frac{2}{x}\left(1-q^{n+1}\right) J_{n+1}(x, a ; q)-q^{n+1} J_{n+2}(x, a ; q) ; \quad \forall a=0,2,4, \ldots
$$

which is the recurrence relation of each of $J_{n}^{(1)}(x ; q)$ and $J_{n}^{(2)}(x ; q)$.

Case (2): The function $J_{n}(x, a ; q)$ has the recurrence relation

$$
\begin{aligned}
J_{n}(x, a ; q)= & q^{\frac{n}{4}}\left[\frac{2}{x}\left(1-q^{n+1}\right)-q^{\frac{n}{2}} \frac{x}{2}\right] J_{n+1}(x, a ; q) \\
& +q^{n+1 / 2} J_{n+2}(x, a ; q) ; \quad \forall a=1,3,5, \ldots
\end{aligned}
$$

which is the recurrence relation of each of $J_{n}^{(3)}(x ; q)$ and $J_{n}^{(4)}(x ; q)$.

\section{The quantum algebra approach to $J_{n}(x, a ; q)$}

The quantum algebra $E_{q}(2)$ is determined by generators $H, E_{+}$and $E_{-}$with the commutation relations

$$
\left[H, E_{+}\right]=E_{+}, \quad\left[H, E_{-}\right]=-E_{-}, \quad\left[E_{-}, E_{+}\right]=0 .
$$

By considering the irreducible representations $(\omega)$ of $E_{q}(2)$ characterized by $\omega \in \mathbb{C}$, then the spectrum of the operator $H$ will be the set of integers $\mathbb{Z}$, and the basis vectors $f_{m}, m \in \mathbb{Z}$, satisfy

$$
E_{ \pm} f_{m}=\omega f_{m \pm 1}, \quad H f_{m}=m f_{m}, \quad E_{+} E_{-} f_{m}=\omega^{2} f_{m}
$$

where $C=E_{+} E_{-}$is the Casimir operator which commutes with the generators $H, E_{+}$ and $E_{-}$. The following differential operators presented a simple realization of $(\omega)$

$$
H=z \frac{d}{d z}, \quad E_{+}=\omega z, \quad E_{-}=\frac{\omega}{z}
$$

acting on the space of all linear combinations of the functions $z^{m}, z$ a complex variable, $m \in \mathbb{Z}$, with basis vectors $f_{m}(z)=z^{m}$. 
In the ordinary Lie theory, matrix elements $T_{s m}$ of the complex motion group in the representation $(\omega)$ are typically defined by the expansions [7-9]

$$
e^{\alpha E_{+}} e^{\beta E_{-}} e^{\tau H} f_{m}=\sum_{s=-\infty}^{\infty} T_{s m}(\alpha, \beta, \tau) f_{s}
$$

If we replace the mapping $e^{x}$ by the mapping $E_{q}(x, a / 2)$ from the Lie algebra to the Lie group with putting $\tau=0$ in equation (42), we can use the model (41) to find the following $q$-analog of matrix elements of $(\omega)$ :

$$
E_{q}\left(\alpha E_{+}, a / 2\right) E_{q}\left(\beta E_{-}, a / 2\right) f_{m}=\sum_{s=-\infty}^{\infty} T_{s m}(\alpha, \beta) f_{s}
$$

and hence

$$
E_{q}\left(\alpha \omega z, \frac{a}{2}\right) E_{q}\left(\beta \frac{\omega}{z}, \frac{a}{2}\right) z^{m}=\sum_{r, t=0}^{\infty} \frac{q^{\frac{a}{2}\left[\left(\begin{array}{l}
r \\
2
\end{array}\right)+\left(\begin{array}{l}
t \\
2
\end{array}\right)\right]}}{(q ; q)_{r}(q ; q)_{t}} \omega^{r+t} \alpha^{r} \beta^{t} z^{m-t+r} .
$$

Now replace $s$ by $m-t+r$ to get

$$
E_{q}\left(\alpha \omega z, \frac{a}{2}\right) E_{q}\left(\beta \frac{\omega}{z}, \frac{a}{2}\right) z^{m}=\sum_{s=-\infty}^{\infty} \sum_{r=0}^{\infty} \frac{q^{\frac{a}{2}\left[\left(\begin{array}{c}
r \\
2
\end{array}\right)+\left(\begin{array}{c}
m+r-s \\
2
\end{array}\right)\right]}}{(q ; q)_{r}(q ; q)_{m+r-s}} \omega^{m+2 r-s} \alpha^{r} \beta^{m+r-s} z^{s}
$$

and by equating the coefficient of $z^{s}$ for $m \geq s$ on both sides, we get

$$
\begin{aligned}
T_{s m}(\alpha, \beta)= & \frac{q^{\frac{a}{2}\left(\begin{array}{c}
m-s \\
2
\end{array}\right)}}{(q ; q)_{m-s}}(\omega \beta)^{m-s} \sum_{r=0}^{\infty} \frac{q^{\frac{a}{2} r(r+m-s-1)}}{(q ; q)_{r}\left(q^{m-s+1} ; q\right)_{r}}\left(\omega^{2} \alpha \beta\right)^{r}, \quad m \geq s \\
= & q^{\frac{a}{4}(m-s)^{2}}\left((-1)^{-(a+1)} \frac{\beta}{\alpha}\right)^{\frac{m-s}{2}}(-1)^{(m-s)(a+1)} J_{m-s}\left(2 q^{\frac{-a}{4}} \omega \sqrt{(-1)^{a+1} \alpha \beta}, a ; q\right), \\
& (-1)^{a+1} \alpha \beta>0 ; m \geq s \\
= & q^{\frac{a}{4}(s-m)^{2}}\left((-1)^{(a+1)} \frac{\alpha}{\beta}\right)^{\frac{s-m}{2}} J_{s-m}\left(2 q^{\frac{-a}{4}} \omega \sqrt{(-1)^{a+1} \alpha \beta}, a ; q\right), \\
& (-1)^{a+1} \alpha \beta>0 ; m \geq s,
\end{aligned}
$$

where $J_{-n}(x, a ; q)=(-1)^{n(a+1)} J_{n}(x, a ; q)$.

Similarly,

$$
\begin{aligned}
& T_{s m}(\alpha, \beta)=q^{\frac{a}{4}(s-m)^{2}}\left((-1)^{(a+1)} \frac{\alpha}{\beta}\right)^{\frac{s-m}{2}} J_{s-m}\left(2 q^{\frac{-a}{4}} \omega \sqrt{(-1)^{a+1} \alpha \beta}, a ; q\right), \\
& (-1)^{a+1} \alpha \beta>0 ; s \geq m .
\end{aligned}
$$

The combination between the two cases gives us the following expression:

$$
\begin{aligned}
& T_{s m}(\alpha, \beta)=q^{\frac{a}{4}(s-m)^{2}}\left((-1)^{(a+1)} \frac{\alpha}{\beta}\right)^{\frac{s-m}{2}} J_{s-m}\left(2 q^{\frac{-a}{4}} \omega \sqrt{(-1)^{a+1} \alpha \beta}, a ; q\right), \\
& (-1)^{a+1} \alpha \beta>0
\end{aligned}
$$

which is valid for all $m, s \in \mathbb{Z}$. Then we get the following result. 


\section{Lemma 5.1}

$$
\begin{aligned}
& E_{q}\left(\alpha \omega z, \frac{a}{2}\right) E_{q}\left(\beta \frac{\omega}{z}, \frac{a}{2}\right) \\
& \quad=\sum_{s=-\infty}^{\infty} q^{\frac{a}{4} s^{2}}\left((-1)^{(a+1)} \frac{\alpha}{\beta}\right)^{\frac{s}{2}} J_{s}\left(2 q^{\frac{-a}{4}} \omega \sqrt{(-1)^{a+1} \alpha \beta}, a ; q\right) z^{s},
\end{aligned}
$$

where $(-1)^{a+1} \alpha \beta>0$.

As special cases:

Considering (45) with $a=0, \alpha=-\beta=1, z=t$ and $\omega=\frac{x}{2}$, we obtain the relation (16).

Considering (45) with $a=2, \alpha=-\beta=1, z=\frac{t}{\sqrt{q}}$ and $\omega=\frac{\sqrt{q} x}{2}$, we obtain the relation (17).

Considering (45) with $a=1, \alpha=\beta=1, z=t$ and $\omega=\frac{\sqrt[4]{q} x}{2}$, we obtain the relation (18).

Considering (45) with $a=3, \alpha=\beta=1, z=t$ and $\omega=\frac{q^{3 / 4} x}{2}$, we obtain the relation (19).

\section{Competing interests}

The author declares that he has no competing interests.

\section{Author's contributions}

The author read and approved the final manuscript.

Received: 23 February 2013 Accepted: 15 April 2013 Published: 29 April 2013

\section{References}

1. Gasper, G, Rahman, M: Basic Hypergeometric Series, 2nd edn. Cambridge University Press, Cambridge (2004)

2. Floreanini, R, LeTourneux, J, Vinet, L: More on the $q$-oscillator algebra and q-orthogonal polynomials. J. Phys. A, Math. Gen. 28, L287-L293 (1995)

3. Exton, H: q-Hypergeometric Functions and Applications. Ellis Horwood, Chichester (1983)

4. Atakishiyev, NM: On a one-parameter family of q-exponential functions. J. Phys. A, Math. Gen. 29, L223-L227 (1996)

5. Jackson, FH: On q-functions and certain difference operator. Trans. R. Soc. Edinb. 46, 253-281 (1908)

6. Jackson, FH: The application of basic numbers to Bessel's and Legendre's functions. Proc. Lond. Math. Soc. 2, 192-220 (1903-1904)

7. Rogov, V-BK: q-Bessel Macdonald functions. arXiv:math.QA/0010170v1, 17 Oct. 2000

8. Rogov, V-BK: The integral representations of the $q$-Bessel Macdonald functions. arXiv:math.QA/0101259v1, 31 Jan. 2001

9. Erdélyi, A, Magnus, W, Oberhettinger, F, Tricomi, FG: Higher Transcendental Functions, vol. 2. McGraw-Hill, New York (1953) (based, in part, on notes left by Harry Bateman)

10. Mansour, M, Al-Shomarani, MM: New $q$-analogy of modified Bessel function and the quantum algebra $E_{q}(2)$. J. Comput. Anal. Appl. 15(4), 655-664 (2013)

11. Miller, W: Lie Theory and Special Functions. Academic Press, San Diego (1968)

12. Kalnins, EG, Mukherjee, S, Miller, W: Models of $q$-algebra representations: the group of plane motions. SIAM J. Math. Anal. 25, 513-527 (1994)

13. Dattoli, G, Torre, A: $q$-Bessel functions: the point of view of the generating function method. Rend. Mat. Appl. (7) 17, 329-345 (1997) 